\title{
Lethally high ROS levels thwart resistance
}

Many strategies have been proposed to eradicate cancer cells that have become resistant to targeted therapies. Despite substantial research in this area, relapse owing to the emergence of drug resistant cells occurs in most patients. A recent study has identified an acquired vulnerability to high levels of reactive oxygen species (ROS) in $B R A F^{\mathrm{V} 600 \mathrm{E}_{-}}$ mutant melanomas that are resistant to BRAF and MEK inhibitors.

Wang, Leite de Oliveira et al. generated BRAF inhibitor-resistant (resistant) or BRAF and MEK inhibitor-resistant (double-resistant) cells from the human melanoma cell lines A375 and Mel888. Resistance in these cells was acquired through expression of different resistance mutations commonly seen in patients, and withdrawal of drugs impaired proliferation of resistant and double-resistant cells, mimicking the 'drug holiday' effect observed in the clinic. These results suggest that the cells are a reasonable model for clinical resistance.

Increased RAS signalling is known to increase ROS production, and indeed ROS levels in resistant and double-resistant cells were substantially higher than those in parental cells. Further induction of ROS in these resistant cells using the chemical paraquat induced death via apoptosis but only mildly impaired proliferation of parental cells.

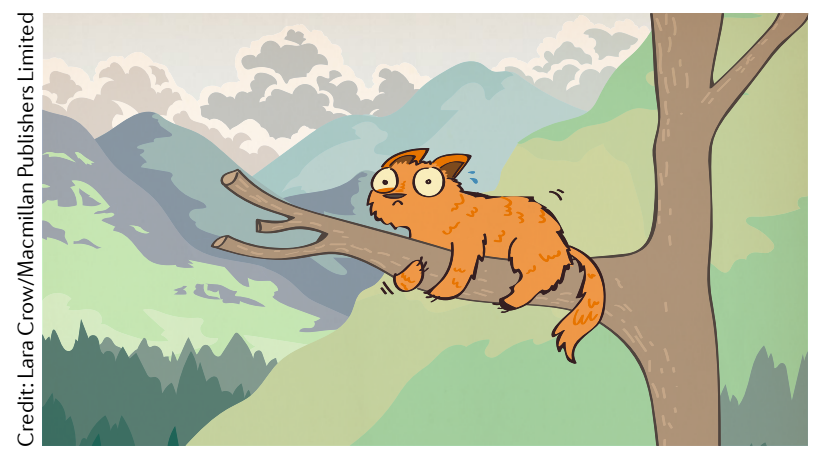

To apply this finding clinically, the authors looked for drugs that can induce ROS and identified the histone deacetylase inhibitor (HDACi) vorinostat. Vorinostat had a similar effect on cells as paraquat, and this was prevented by treatment with a ROS scavenger. Interestingly, MEK inhibitor-resistant NRAS-mutant melanoma cells were also sensitive to vorinostat, suggesting that this treatment might be broadly applicable across melanomas, regardless of the initial MAPK pathway mutation.

Looking into the mechanism of action of ROS induction by vorinostat, the authors identified reduced expression of SLC7A11, which encodes a transporter that permits cellular uptake of cystine, the precursor of the antioxidant glutathione. SLC7A11 suppression in resistant and double-resistant melanoma cells was sufficient to increase ROS levels, and SLC7A11 overexpression rescued HDACi-mediated ROS induction.

There is some evidence that combined HDAC and MAPK inhibitor treatment can prevent the emergence of resistance; however, other evidence suggests that ROS can activate the MAPK pathway. In the resistant and double-resistant cells, the combination of an HDACi and a BRAF or MEK inhibitor did not prevent proliferation of resistant cells, indicating that simultaneous treatment is not likely to be beneficial. Therefore, the authors hypothesized that sequential treatment was most likely to be therapeutically beneficial and tested such a regimen in mice.

Immunodeficient mice were engrafted with A375 cells, and mice with established tumours were treated with a BRAF inhibitor (PLX4720). Following the emergence of resistance, mice were randomized to receive one of four treatments.
Continuous PLX4720 treatment resulted in rapid tumour growth, whereas treatment with no drug produced a drug holiday effect (no growth followed by slow growth). Treatment with PLX4720 and vorinostat mimicked the drug holiday effect, consistent with an antagonistic effect of these inhibitors. Treatment with vorinostat alone significantly reduced tumour volumes, consistent with in vitro data.

The authors initiated a small clinical proof-of-concept study to test the effects of vorinostat (which has been approved for lymphoma) in patients with double-resistant $B R A F^{\mathrm{V} 600 \mathrm{E}}$-mutant melanoma. To do this rapidly, they synthesized vorinostat under good manufacturing practice conditions. So far, 6 patients have been treated, and molecular data from 3 patients indicate that vorinostat repressed SLC7A11 expression in lesions. It also reduced the allele frequency of BRAF and MEK inhibitor resistance mutations, indicating that resistant cells have been eliminated.

Although patient efficacy data are not reported, the authors note in their discussion that tumours initially stabilize and then progress as residual non-resistant cells grow out. As such, the authors are adapting the clinical trial to monitor for emerging resistance (using cell free DNA in the blood) and use pulsatile vorinostat treatment to eliminate resistant cells before switching back to BRAF and MEK inhibitor therapy.

Overall, this work raises several important considerations for developing therapies that effectively thwart resistance and for rapidly translating preclinical data.

Sarah Seton-Rogers

ORIGINAL ARTICLE Wang, L., Leite de Oliveira, R. et al. An acquired vulnerability of drug-resistant melanoma with therapeutic potential. Cell https:// doi.org/10.1016/j.cell.2018.04.012 (2018) 